\title{
Direct generation of radially-polarized output from an Yb-doped fiber laser
}

\author{
D. Lin, J. M. O. Daniel, M. Gecevičius, M. Beresna, P. G. Kazansky, W. A. Clarkson \\ Optoelectronics Research Center, University of Southampton, Southampton, SO17 1BJ, UK \\ dl1n10@soton.ac.uk
}

\begin{abstract}
A simple technique for directly generating a radially-polarized output beam from an ytterbium-doped fiber laser using an intracavity S-waveplate is reported. The laser yielded $7 \mathrm{~W}$ of output with a corresponding slope efficiency of $67 \%$.

OCIS codes: (140.3510) Laser, fiber; (260.5430) Polarization; (050.6624) Subwavelength structures
\end{abstract}

\section{Introduction}

Radially-polarized light is characterized by a donut-shaped intensity profile and exhibits axially symmetry both in field-amplitude and polarization direction. Radially-polarized beams can produce a strong longitudinal electric field component along the beam axis under the condition of tight focusing [1]. This unique property leads to a range of promising applications in material processing, high-resolution microscopy, particle acceleration and trapping.

Fiber lasers benefit from a geometry that is advantageous for scaling average power and hence could be a promising route to high power radially-polarized beams. Indeed, several recent investigations were conducted in ytterbium-doped and erbium-doped fiber lasers by employing either a birefringent crystal aligned for propagation along the optic axis [2], a segmented wave plate [3] or a photonic crystal grating[4]. However, these schemes produced rather low output powers or had relatively low efficiency. The highest laser power and slope efficiency reported to date for a fiber lasers with a radially-polarized output is $2.5 \mathrm{~W}$ and $46 \%$ respectively [4], which is far below the performance reported for bulk solid-state lasers.

Here, we present an alternative approach for efficiently generating radially-polarized output directly in an $\mathrm{Yb}$ doped fiber laser by employing a S-waveplate in an external feedback cavity arrangement. The S-waveplate consists of spatially-variant sub-wavelength gratings which are produced by femtosecond laser pulse direct writing in a fused-silica window. These grating structures induce form birefringence with slow and fast axes aligned parallel and perpendicular to the grating direction respectively, which is aligned at an angle $\varphi / 2$ from the azimuthal angle $\varphi$ [5].

\section{Experiment and Results}

The experiment set-up, illustrated in Fig. 1, comprised a $2 \mathrm{~m}$ length of non-polarization maintaining Yb-doped fiber with an external feedback cavity. The fiber had a core diameter of $20 \mu \mathrm{m}$, numerical aperture (NA) of 0.07 , and an inner-cladding diameter of $125 \mu \mathrm{m}$. The corresponding V-number is approximately 4.07 guaranteeing that only the fundamental $\mathrm{LP}_{01}$ mode and its neighboring $\mathrm{LP}_{11}$ modes could be guided within the fiber. The end of fiber adjacent to the external cavity was cleaved at $8^{\circ}$ to suppress the broadband feedback and hence parasitic lasing between the fiber end facets. The opposite end was perpendicularly-cleaved to serve as the output coupler. Pump light was provided by a fiber-coupled $976 \mathrm{~nm}$ laser diode (LD) with a $105 \mu \mathrm{m}$ core diameter and $0.12 \mathrm{NA}$, which was freespace coupled into the output end of the fiber via a dichroic mirror. The light emerging from the angle-cleaved end of fiber was collimated by an aspheric lens and feedback for lasing was provided by a plane high reflectivity mirror at $1.06 \mu \mathrm{m}$. A S-waveplate and a polarization beam splitter (PBS) were placed in the external cavity between rear mirror and fiber collimating lens. The PBS allowed only p-polarized light to pass through it and then transmit to the $\mathrm{S}$-waveplate. When the orientation of the S-waveplate, shown by the arrow in Fig. 1(a), is aligned parallel to the ppolarization, it converts the p-polarized beam into radially-polarized light, which is then coupled into the fiber to excite the radially-polarized $\mathrm{TM}_{01}$ mode.

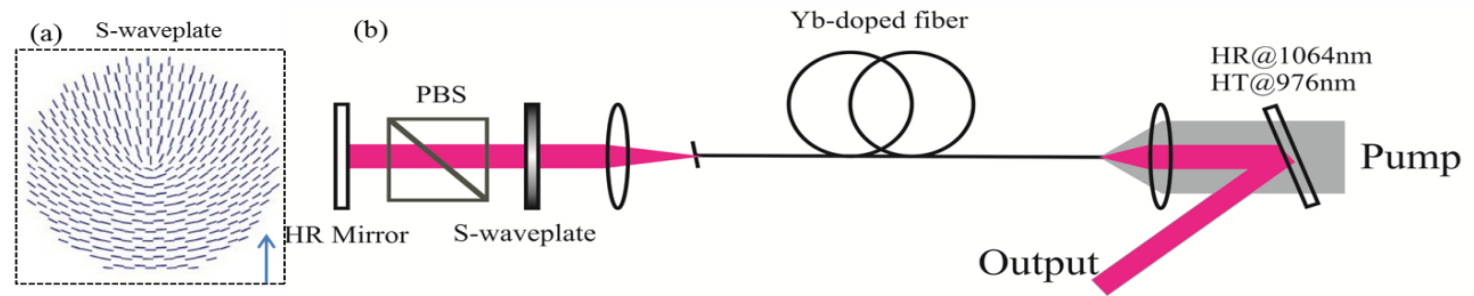

Fig. 1 (a) Schematic of nanogratings orientation in SVPC, (b) Experimental laser set-up. 
The typical output beam intensity distribution at the maximum output power is shown in Fig. 2 (a). Fig. 2 (b)-(d) show the two-lobe structures of beam after passing through a polarizer oriented at different angles verifying its radial polarization. The polarization purity was measured to be $87 \%$ according to the method described in [4].
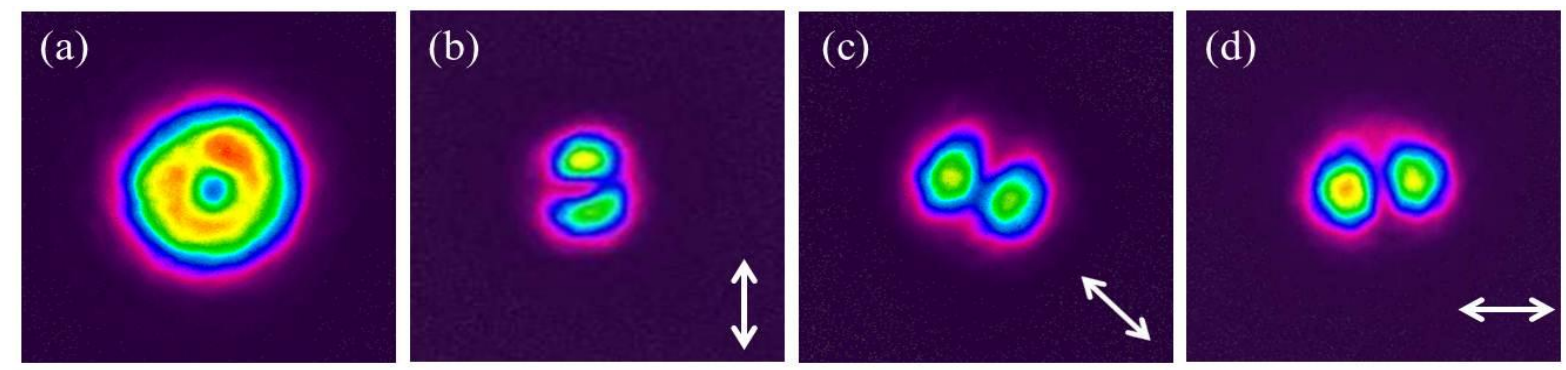

Fig. 2 Experimentally measured beam intensity distribution (a) without polarizer, (b) with polarizer at $90^{\circ}$, (c) with polarizer at $45^{\circ}$, (d) with polarizer at $0^{\circ}$.

The one-dimensional intensity distribution across the center of beam in Fig. 2 (a) is shown in Fig. 3. There is residual intensity at the center of the beam indicating that the fundamental $\mathrm{LP}_{01}$ mode was also excited. However, the power ratio of the $\mathrm{LP}_{01}$ to $\mathrm{TM}_{01}$ mode is approximately 1:2.5 confirming that most of the power is in the radiallypolarized mode. Parasitic laser oscillation on the $\mathrm{LP}_{01}$ mode can be attributed to the high-doping density of $\mathrm{Yb}$ ions at the center of fiber core resulting in a higher single pass gain for the $\mathrm{LP}_{01}$ mode than for $\mathrm{TM}_{01}$ mode in the fiber. This could be suppressed by using a better tailored core design with a dip in the $\mathrm{Yb}$ concentration at the center of the fiber. The laser output power as a function of incident pump power is shown in Fig. 4. The laser reached threshold at a pump power of $1.5 \mathrm{~W}$ and yielded $7 \mathrm{~W}$ of output at the maximum incident pump power of $12 \mathrm{~W}$ with a corresponding slope efficiency of $67 \%$. The beam propagation factor $\left(\mathrm{M}^{2}\right)$ was measured to be 1.7 , which is slightly less than theoretical value of 2 due to the presence of the fundamental $\mathrm{LP}_{01}$ mode.

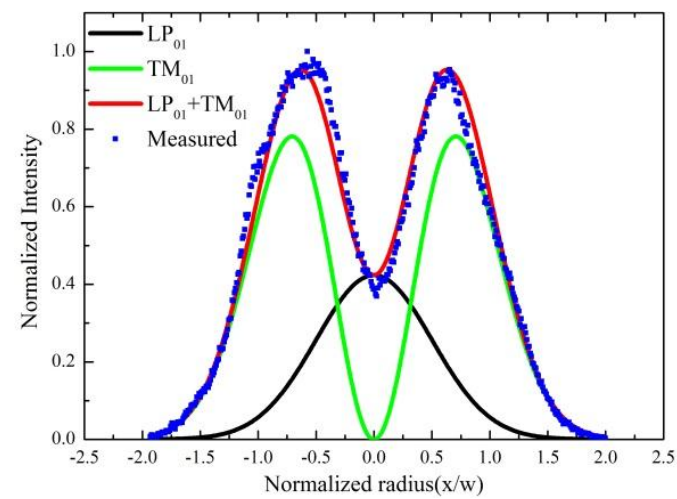

Fig. 3 The calculated and measured transverse intensity distribution of output beam.

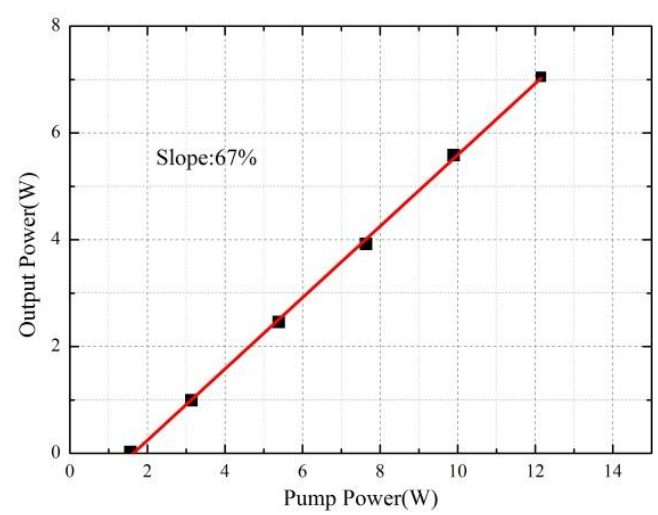

Fig. 4 Laser output Power versus pump power

\section{Conclusion}

In summary, a fiber laser with an intracavity S-waveplate has been described which efficiently generates a donutshaped output beam with radial polarization. A maximum power of $7 \mathrm{~W}$ was obtained with a slope efficieny of $67 \%$. To the best of our knowledge, this is the highest power and highest slope efficiency for radially-polarized fiber laser to date. The prospects for further improvement in performance will be discussed.

\section{References}

[1] R. Dorn, S. Quabis, and G. Leuchs, "Sharper Focus for a Radially Polarized Light Beam," Physical Review Letters 91 (2003).

[2] R. Zhou, B. Ibarra-Escamilla, J. W. Haus, P. E. Powers, and Q. Zhan, "Fiber laser generating switchable radially and azimuthally polarized beams with $140 \mathrm{~mW}$ output power at $1.6 \mu \mathrm{m}$ wavelength," Applied Physics Letters 95, 191111 (2009).

[3] M. Fridman, G. Machavariani, N. Davidson, and A. A. Friesem, "Fiber lasers generating radially and azimuthally polarized light," Applied Physics Letters 93 (2008).

[4] D. Lin, K. G. Xia, J. L. Li, R. X. Li, K. Ueda, G. Q. Li, and X. J. Li, "Efficient, high-power, and radially polarized fiber laser," Optics Letters $35,2290-2292(2010)$.

[5] M. Beresna, M. Gecevicius, and P. G. Kazansky, "Polarization sensitive elements fabricated by femtosecond laser nanostructuring of glass [Invited]," Opt Mater Express 1, 783-795 (2011). 